\title{
Repères \\ sur la gestion et les finances publiques En Décembre 2021
}

\section{BUDGET DE L'ÉTAT ET DES OPÉRATEURS}

\section{$\pi$ Procédure budgétaire}

Loi du $1^{\text {er }}$ décembre 2021 de finances rectificative pour 2021

Cette seconde loi de finances rectificative pour 2021 a été promulguée le 1er décembre 2021 et publiée au Journal officiel du 2 décembre 2021. Elle permet de financer "l'indemnité inflation » de 100 euros pour soutenir les ménages face à la hausse des prix ainsi qu'un complément de 100 euros au "chèque énergie » pour les ménages les plus modestes. Le contenu de ce texte avait été présenté dans les Repères de novembre.

Loi organique du 28 décembre 2021 relative à la modernisation de la gestion des finances publiques

Cette loi organique a été promulguée le 28 décembre 2021 et publiée au Journal officiel du 29 décembre 2021. Dans sa décision du 23 décembre, le Conseil constitutionnel avait validé les grandes lignes de ce texte. II réforme le cadre des finances publiques, 20 ans après I'adoption de la loi organique du 1er août 2001 relative aux lois de finances (LOLF).

Les dispositions de cette loi s'articulent autour de trois grands axes :

- Le renforcement du pilotage pluriannuel des finances publiques ;

- L'amélioration de la transparence des finances publiques ;

- La rationalisation et l'amélioration du travail parlementaire.

La plupart de ses dispositions seront applicables aux lois de finances portant sur l'année 2023.

La loi organique prévoit que les lois de programmation des finances publiques (LPFP) et les lois de finances définiront à l'avenir les ob- jectifs de dépenses publiques en Mds€, plutôt qu'en points de produit intérieur brut (PIB), avec une évolution en pourcentage. Les projets annuels de performances (PAP), qui présentent pour les ministères l'ensemble des moyens regroupés au sein d'une mission et alloués à une politique publique, devront se doter d'une trajectoire de performance triennale. En amont du dépôt du projet de loi de finances de l'année, le gouvernement devra justifier devant le Haut Conseil aux finances publiques (HCFP) les éventuels écarts par rapport à la trajectoire pluriannuelle de la LPFP.

Aussi, un véritable "temps des finances locales " sera consacré au sein de la discussion budgétaire, avec un rapport sur la situation d'ensemble des finances publiques locales et un débat de synthèse préalable au projet de loi de finances. Le contenu de ce rapport a été enrichi par les sénateurs afin de nourrir le débat parlementaire, en particulier sur les charges transférées aux collectivités territoriales.

Le texte crée une nouvelle catégorie de lois de finances : la «loi de finances de fin de gestion» qui ne pourra comporter aucune mesure fiscale nouvelle. Cette disposition pérennise la pratique des collectifs budgétaires de fin d'année suivie par le gouvernement depuis 2018.

Le calendrier budgétaire est rationalisé. Le débat relatif au programme de stabilité (fin avril) et le débat d'orientation des finances publiques (début juillet) sont fusionnés en une séquence spécifique dédiée à l'orientation pluriannuelle des finances publiques, qui se déroulera au printemps. À cette occasion, le gouvernement devra présenter un rapport annuel sur l'évolution de l'économie nationale et sur les orientations des finances publiques. Ce rapport comprendra également des éléments sur la situation des administrations publiques locales.

La date limite de dépôt du projet de loi de règlement est avancée du 1er juin au 1er mai afin de donner plus de temps aux parlementaires 
pour se consacrer à leurs travaux d'évaluation et de contrôle dans le cadre du « printemps de l'évaluation ", pratique observée depuis quatre ans à l'Assemblée nationale et qui est consacrée dans la LOLF.

Les lois de règlement sont renommées "lois relatives aux résultats de la gestion et portant approbation des comptes de l'année " afin de mettre en avant l'importance des travaux liés à l'évaluation des politiques publiques à l'occasion de leur examen.

Un nouveau temps parlementaire dédié au sujet de la dette publique est instauré avant le début de la session ordinaire, à l'automne. Le gouvernement devra transmettre un rapport sur la trajectoire, les conditions de financement et la soutenabilité de la dette publique, qui pourra donner lieu à débat.

Enfin, la loi organique étend les avis du HCFP à la " cohérence » de l'article liminaire des projets de lois de finances et de financement de la sécurité sociale au regard de l'objectif pluriannuel de dépenses fixé dans la LPFP et des prévisions de recettes, de dépenses et de solde des projets de lois au regard des prévisions économiques connues. Plusieurs dispositions non organiques sur le HCFP font l'objet d'une loi ordinaire du 6 décembre 2021 portant diverses dispositions relatives au Haut Conseil des finances publiques et à l'information du Parlement sur les finances publiques.

\section{Loi de finances pour 2022}

La loi de finances pour 2022 a été promulguée le 30 décembre 2021 et été publiée au Journal officiel du 31 décembre 2021. Dans une décision du 28 décembre 2021, le Conseil constitutionnel avait validé l'essentiel des dispositions de cette loi.

Le budget 2022 prolonge la mise en œuvre du "Plan de relance», avec notamment le renforcement de mesures pour l'emploi.

Il poursuit et accélère la transition écologique. Le dispositif de rénovation énergétique des logements, MaPrimRénov', est notamment prolongé en 2022.

Plusieurs priorités gouvernementales à destination des jeunes sont également traduites : poursuite du développement du service national universel et du service civique ou encore prolongation jusqu'en juin 2022 des primes pour le recrutement d'un apprenti.

Des amendements du gouvernement sont venus compléter le texte afin d'inscrire deux mesures annoncées le 12 juillet dernier par le président de la République :

- Le plan d'investissement pour bâtir la France de 2030, à hauteur de $34 \mathrm{Mds} €$, dont 3,5 Mds€ de crédits dès 2022 ;

- La création d'un revenu d'engagement pour les jeunes, destiné aux jeunes de moins de 26 ans sans emploi ou formation. Plus de 500 millions ont été votés pour la mise en $œ u v r e$ de ce nouveau revenu qui doit débuter le $1^{\text {er }}$ mars 2022. Ses bénéficiaires percevront une allocation allant jusqu'à 500 euros par mois, en contrepartie de 15 à 20 heures de formation ou d'accompagnement par semaine, sur une durée de 6 à 12 mois.

Aussi, un «bouclier tarifaire » est mis en place pour contenir la forte hausse du prix du gaz et du prix de l'électricité. Le bouclier s'appliquera

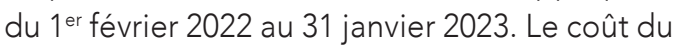
bouclier est, à ce stade, prévu à 5,9 Mds€.

La péréquation régionale est réformée. Pour soutenir la relance de la construction de logements sociaux, l'État s'engage à garantir aux collectivités une compensation intégrale pendant dix ans de la perte de recettes liée à l'exonération de taxe foncière sur les propriétés bâties (TFPB) pour les nouvelles constructions.

Conformément au discours du chef de l'État du 2 septembre dernier, des amendements du gouvernement financent le plan « Marseille en grand " (rénovation de 174 écoles de la ville, $1 \mathrm{Md} €$ pour les transports prioritaires de la métropole Aix-Marseille-Provence).

Les baisses d'impôts des particuliers et des entreprises, décidées avant la crise sanitaire, sont maintenues. La taxe d'habitation des $20 \%$ des ménages les plus aisés est encore réduite, après une première étape en 2021. Ces ménages bénéficient en 2022 d'une exonération de $65 \%$ de leur taxe. La taxe d'habitation sur les résidences principales sera supprimée pour tous les contribuables en 2023.

Le taux normal de l'impôt sur les sociétés passe à $25 \%$ pour toutes les entreprises en 2022. II s'agit de la dernière étape de la réforme engagée en 2018, dans le but d'améliorer leur compétitivité.

Par ailleurs, les députés ont adopté la défiscalisation et la désocialisation en 2022 et 2023 des pourboires reçus par les salariés de tous les secteurs d'activité, rémunérés en deçà de 1,6 Smic.

Les moyens des ministères régaliens sont augmentés en 2022. Les crédits du ministère de la défense, comme le prévoit la loi de program- 
mation militaire, sont en hausse. Le budget du ministère de l'intérieur augmente également pour mettre en œuvre les mesures annoncées à I'issue du "Beauvau de la sécurité ». En outre, comme en 2021, aucune suppression d'emplois n'interviendra dans le réseau territorial des préfectures et sous-préfectures. Le budget de la justice progresse également, pour la deuxième année consécutive, de $8 \%$. L'éducation nationale, premier poste du budget de l'État, bénéficie en 2022 d'une rallonge de 1,7 Md€, notamment pour financer les revalorisations salariales des enseignants. L'investissement dans la recherche est accentué (+760 M€). Dans le même temps, les effectifs des emplois publics de l'État et de ses opérateurs restent à un niveau quasi-stable depuis 2017.

Enfin, par cette loi de finances, le gouvernement est autorisé à légiférer par ordonnance pour réformer la justice financière et la responsabilité des gestionnaires publics. Cette réforme interviendra au plus tard le $1^{\text {er }}$ janvier 2023.

\section{$\lambda$ Politique budgétaire}

La politique de développement des biocarburants

Dans un rapport publié le 20 décembre 2021, la Cour des comptes a présenté quelques observations sur la politique de développement des biocarburants. Les biocarburants sont des substituts aux carburants fossiles auxquels ils sont incorporés. La France a choisi d'encourager leur incorporation dans des proportions de plus en plus élevées, ce qui a eu des effets significatifs: le taux d'énergie renouvelable dans les transports a atteint $9,25 \%$ en 2019 , soit un pourcentage élevé au sein de l'UE, et notre pays est le premier producteur européen de biodiesel et le deuxième d'éthanol. Cependant, les productions de biocarburants conventionnels, disponibles à la pompe, plafonnent depuis une dizaine d'années, et le solde correspondant des échanges commerciaux est déficitaire depuis 2016 - ce qui limite les bénéfices de cette politique pour les agriculteurs. En outre, de nombreuses études scientifiques concluent au bilan environnemental défavorable des biocarburants conventionnels et à une réduction limitée des émissions de gaz à effet de serre.

Aussi, la fiscalité est le principal outil mis en œuvre par l'État pour favoriser la consommation de biocarburants. Or, la Cour constate que les réductions des tarifs de taxe intérieure de consommation sur les produits énergétiques (TICPE) pour les carburants à fort taux d'incor- poration sont appliquées sans aucune rationalité à certains d'entre eux et sans tenir compte des surcoûts de production imputables aux biocarburants incorporés. Elles conduisent à une surcompensation de ces surcoûts, ce qui n'est pas conforme à la règlementation européenne sur la taxation des produits énergétiques. Sur ce point précis, la Cour recommande de revoir les réductions de TICPE pour qu'elles soient désormais accordées au regard d'éléments fiables et objectifs concernant les surcoûts de production.

\section{$\lambda$ Budget des opérateurs}

La gestion du Commissariat à l'énergie atomique et aux énergies alternatives (CEA) et ses grands projets (2013-2019)

Dans un rapport publié le 15 décembre 2021, la Cour des comptes présente une analyse de la gestion et du financement du CEA.

Doté d'un budget de 5,3 Md€ et disposant d'un effectif de 20691 salariés, répartis sur neuf centres dans quatre régions, le CEA est I'un des trois plus grands opérateurs de l'État. Organisme de recherche classé comme établissement public à caractère industriel et commercial (EPIC), le CEA a récemment dû faire face à de nombreuses difficultés dans la conduite de certaines de ses activités nucléaires. Le CEA a vu progressivement son domaine de compétence élargi à la recherche technologique pour l'industrie, aux sciences de la matière et de l'univers, à la santé et aux sciences du vivant et enfin aux énergies renouvelables, au climat et à l'environnement - quand il était à l'origine exclusivement acteur de la recherche et du développement de l'énergie nucléaire. La Cour souligne que le renforcement de ses fonctions transversales conditionnera sa capacité à les conduire au mieux. En outre, la mutation entamée depuis une dizaine d'années doit se poursuivre, en garantissant à l'avenir la soutenabilité de son modèle. Dans le présent rapport, la Cour formule au total 12 recommandations.

Concernant en particulier le pilotage stratégique de l'État, deux outils principaux sont mis en œuvre : le contrat d'objectifs et de performance (COP), conclu tous les cinq ans avec l'État, qui fixe les objectifs pour la période et détermine les indicateurs de mesurer des résultats, et le plan à moyen et long terme (PMLT), document annuel couvrant une période glissante de 10 ans, qui détaille, par programme, les résultats attendus et les moyens financiers et humains nécessaires pour les atteindre. Ces documents, dont l'établissement comporte un 
exercice indispensable de programmation pluriannuelle, ne sont pas accompagnés d'engagement financier de l'État. Un tel engagement, rééquilibrant les obligations des deux parties, serait de nature à davantage responsabiliser le CEA et à lui donner une prévisibilité accrue.

Selon la Cour, malgré les efforts importants déjà réalisés, la soutenabilité du modèle économique du CEA, à moyen terme, dépend pour beaucoup de la levée des incertitudes relatives aux grands projets, ainsi que de sa capacité à continuer à maitriser la masse salariale malgré les besoins qui accompagnent les réformes engagées. La mobilisation par l'État de nouveaux crédits exceptionnels, dans du plan de relance notamment, doit avoir pour corollaire la poursuite de la mutation du CEA et une réflexion stratégique approfondie sur le positionnement du CEA comme partenaire des industriels du nucléaire et des énergies renouvelables ainsi que sur son impact en termes de recettes externes.

\section{FinANCES LOCALES}

La loi de finances pour 2022 et ses incidences sur les collectivités territoriales

La loi de finances pour 2022 publiée le 30 décembre 2021, après la décision du Conseil constitutionnel du 28 décembre 2021, apporte un certain nombre d'évolutions au secteur local. Pour cette dernière loi de finances du quinquennat, et après deux réformes fiscales majeures (taxe d'habitation et impôt de production), aucune réforme n'a été engagée et on constate globalement une stabilité des dotations.

Cependant, des ajustements importants méritent quelques précisions. L'article 113 proroge en 2022, au titre des pertes subies en 2021, deux dotations visant à compenser une partie des pertes de recettes tarifaires et de redevances subies en 2020 par les services publics locaux gérés en régie. La compensation liée à la suppression de la taxe d'habitation est ajustée afin de tenir compte des rôles supplémentaires représentant un surplus de l'ordre de plusieurs centaines de millions d'euros. Il en est de même d'un ajustement de la compensation pour les régions pour tenir compte de la suppression de la CVAE et de la baisse mécanique des frais de gestion.

En matière de dotations, la hausse des dotations de péréquation se poursuit. Notons également qu'un accord a été trouvé sur le financement de l'apprentissage. Les associations d'élus et le CNFPT ont accepté la mise en place, à compter de 2022, d'une cotisation spéciale à l'apprentissage fixée, au maximum, à $0,1 \%$ de la masse salariale des collectivités territoriales, perçue par le CNFPT, permettant de financer 50 \% du coût global de formation. La péréquation régionale a également été réformée en profondeur.

Les flux financiers entre budgets des collectivités locales : 37 Md€ en 2020

Dans cette nouvelle étude, l'OFGL étudie les relations financières entre collectivités. Les montants en jeu sont importants. En 2020, ils s'élèvent à 37 Md€ dont 4,2 Mds€ d'échanges entre les budgets principaux et les budgets annexes.

Mais le plus important des flux financiers concerne les reversements de fiscalité avec les attributions de compensation, la dotation de solidarité communautaire, les mécanismes de péréquation et de compensation. Là encore, sur 2020, I'OFGL estime le montant à 24,7 Md€ en 2020. La troisième grande catégorie concerne les subventions et remboursements de frais entre collectivités évalués à $8 \mathrm{Mds} €$ avec notamment "les remboursements de charges dans le cadre de mises à disposition de personnel, versement de subventions au titre d'opérations d'intérêt partagé, opérations d'investissement pour compte de tiers... ».

\section{Bilan DGF Villes de France 2021}

Dans cette étude, Villes de France actualise les données de la DGF pour ses adhérents. Pour mémoire, cette association regroupe 152 villes adhérentes et les intercommunalités associées avec une population moyenne se situant à 34800 habitants (population DGF).

En 2021, le montant de la DGF est stable avec cependant des évolutions internes. Concernant le bloc communal, la dotation de solidarité urbaine (DSU) augmente de $90 \mathrm{ME}$, soit une progression de 3,78\%. La dotation de solidarité rurale (DSR) augmente également de $90 \mathrm{M} €$. La progression est donc de 5,32 \%. L'écrêtement de la part forfaitaire s'est élevé à $142 \mathrm{M} €$, en augmentation d'environ 7,7 M€ par rapport à I'an dernier, en raison principalement de la population.

\section{Regard financier sur les départements}

Dans cette étude, I'Association des départements de France (ADF) constate que les dé- 
penses d'action sociale ont augmenté dans la part des dépenses de fonctionnement des départements. Elles représentaient $47 \%$ en 2001, 58 \% en 2004 et en 2019 elles s'élevaient à environ $62 \%$. Parmi ces dépenses, le RSA occupe une place à part en raison de la hausse significative du nombre de bénéficiaires dans la majorité des départements en 2020, pour partie compensée par des baisses au premier semestre 2021. En complément de cela, les recettes de fonctionnement ont également augmenté avec une évolution interne majeure : les impôts indirects deviennent prépondérants en 2021. II est également intéressant de noter que les DMTO ont globalement bien résisté en 2020 avec cependant des évolutions très hétérogènes selon les collectivités.

Cette étude note une reprise de l'investissement depuis 2018 après une décennie relativement morose en termes de dépenses d'investissement.

\section{FinANCES SOCIALES}

\section{$\lambda$ Situation des comptes sociaux}

\section{Quels enjeux pour une protection sociale soutenable?}

France Stratégie publie depuis 2020 des « cahiers de soutenabilité » qui soumettent différentes politiques publiques au crible du concept de soutenabilité. Le cahier $n^{0} 1$ «Quels enjeux pour une protection sociale soutenable? ", après avoir passé en revue les différents risques pesant sur le système de protection sociale (ex. vieillissement, ralentissement de la croissance, manque de prospective pluriannuelle), pose les questions du financement et de la dette sociale, ainsi que de la capacité du système à se renouveler, sans privilégier de piste particulière. Le cahier $n^{\circ} 5$ « Comment repenser notre système de santé face aux risques nouveaux? " fait le constat des insuffisances actuelles (ex. caractère inégalitaire, structures en silo, défaillances de la gouvernance) et évoque diverses pistes pour faire évoluer le système.

\section{Qui paye le coût du vieillissement ?}

Une note d'analyse de France Stratégie pose la question de la relation entre l'évolution des dépenses sociales bénéficiant à la population âgée de plus de 60 ans et celle de la répartition du financement de cette dépense. Autrement formulé, est-ce que ce sont les actifs qui supportent le coût du vieillissement de la société ? Après avoir rappelé la très forte croissance de la part des dépenses sociales dans le PIB depuis 40 ans ( de $15 \%$ à $33 \%$ ) l'analyse se focalise sur les dépenses sociales qui concernent plus spécifiquement la population âgée (retraites, maladie et perte d'autonomie); il en ressort que le montant de ces dépenses se concentre massivement sur les classes d'âge 60-79 ans et plus de 80 ans, et que ce sont elles qui ont le plus progressé au cours des 40 dernières années (elles ont mobilisé 6 points de PIB supplémentaires sur la période). Pour autant, l'analyse montre que cette croissance est d'abord due à la démographie (augmentation de la part de ces classes d'âge dans la population totale) et que la dépense par tête pour les plus de 60 ans a moins progressé que le PIB. Cette évolution tient à plusieurs facteurs : tout d'abord, la baisse du poids relatif des retraites du fait du recul de l'âge légal et de la désindexation de celles-ci par rapport aux salaires; d'autre part, la mise à contribution accrue des plus âgés dans le financement de la protection sociale, en raison tout particulièrement de la part croissante des impôts et taxes (spécialement la CSG) dans les ressources de celle-ci en substitution aux cotisations sur le travail ; cela se traduit par le fait que c'est pour les plus de 60 ans que le taux d'effort pour le financement de la protection sociale s'est le plus accru depuis 40 ans. Même si la question du poids du transfert intergénérationnel doit être relativisée, le constat de l'importance des transferts à la population âgée en France amène à se demander si celle-ci n'a pas eu un effet d'éviction au profit des dépenses d'avenir (ex. éducation, climat).

Les comptes de la protection sociale pour 2020 enregistrent une progression très sensible des dépenses

La DREES a publié le 15 décembre le rapport sur la protection sociale en France et en Europe en 2020. (NB : les comptes de la protection sociale couvrent un périmètre sensiblement plus large que celui des lois de financement de la SS). En France, du fait de la crise, les dépenses de protection sociale ont augmenté de 7,8\% en 2020, soit un accroissement de $46 \mathrm{Md}$ (35 Md€ de prestations et $11 \mathrm{Md} €$ d'autres dépenses) directement imputable à la pandémie ; pour l'essentiel, la croissance des prestations est due au risque emploi, suivi du risque santé, les autres dépenses étant constituées par les achats de matériels et de dispositifs (ex. masques, tests) et par les mesures en faveur des professionnels de santé. Les ressources de la protection sociale n'ont diminué que de $0,1 \%$, les compensations massives de l'État aux pertes de ressources des 
différents régimes, principalement pour l'activité partielle, ayant neutralisé les pertes; de ce fait, le déficit du compte de la protection sociale a atteint - $51 \mathrm{Md}$. C'est en France que la part des dépenses de protection sociale par rapport au PIB est la plus forte (35\% du PIB et 12080 € par habitant), notre pays se situant au $4^{\text {e }}$ rang en Europe (sur 20 États) pour la dépense par habitant, derrière le Danemark, l'Autriche et l'Allemagne. Tous les États ont connu des évolutions parallèles, la sauvegarde de l'emploi - avec le recours massif au chômage partiel ayant été les principaux objectifs et instruments des gouvernements. De même, les prestations de lutte contre la pauvreté et l'exclusion ont augmenté fortement (+12\% en France, du fait en particulier du RSA). Les dépenses au titre du risque «vieillesse » ont ralenti leur progression, celles des prestations familiales ont diminué, du fait notamment du moindre recours aux gardes d'enfant.

\section{Dette sociale}

Le financement de la CADES sur les marchés internationaux en 2021-2022

Dans un communiqué de presse du 7 décembre, la CADES a fait le point sur ses opérations de financement sur les marchés internationaux en 2021 et 2022. En 2021, la Caisse aura emprunté 39,4 Md€ à moyen et long terme sur les marchés internationaux, avec un taux moyen de financement de 0,61\% au 30/11/2011. Pour 2022, elle prévoit un programme de financement du même ordre. Au 31 décembre 2021, la CADES aura amorti $205 \mathrm{Md} €$ de dette sociale sur les $320 \mathrm{Md}$ tepris depuis sa création, et les programmes de 2021-2022 s'inscrivent dans la trajectoire de reprise des 136 Mds€ prévus par la loi d'août 2020. Le rapport d'activité 2020 de la CADES donne un tableau des conditions d'amortissement de la dette sociale dans le nouveau contexte de la crise sanitaire.

\section{$\pi$ Lois de financement de la Sécurité sociale}

La promulgation de la loi de financement de la Sécurité sociale pour 2022

Après que, dans sa décision du 16 décembre, le Conseil Constitutionnel a fait disjoindre quelques articles qui n'avaient pas leur place dans une LFSS, la loi de financement de la Sécurité sociale pour 2022 a été publiée au JO du 24 décembre 2021. Elle arrête le déficit 2020 des Régimes obligatoires de base et du FSV à -39,7 Md€ et prévoit pour 2021 un solde négatif de -33,7 Md€. Pour 2022, le déficit prévisionnel des ROBSS et du FSV serait ramené à - 21,4 Md€, soit un peu moins que ce qui était prévu initialement dans le PLFSS (- 22,6 Md€ ; voir Repères de septembre). Pour le moyen terme, les prévisions sont moins pessimistes que celles qui figuraient dans la LFSS 2021, puisque le déficit attendu pour 2024-2025 se stabiliserait autour de $-13 \mathrm{Md}$, les déficits les plus importants étant portés par les régimes d'Assurance maladie et de Vieillesse. Le plafond d'emprunt autorisé pour l'ACOSS en 2022 est de $65 \mathrm{Md}$.

\section{Mesures de soutien contre la crise}

La prolongation des aides pour les secteurs qui continuent d'être impactés fortement par la crise

A la suite de l'annonce par le Premier Ministre, le 9 décembre 2021, de la poursuite des restrictions d'activité directes ou indirectes pour certains secteurs, les ministres de l'économie et du travail ont confirmé la prolongation des dispositifs d'aides pour les entreprises de ces secteurs (ex. traiteurs, agences de voyage, événementiel) : Fonds de solidarité, pour lequel les demandes peuvent être faites au titre de la période allant jusqu'au 31 octobre, activité partielle (accessible à présent dès $65 \%$ de perte de chiffre d'affaires). S'agissant plus particulièrement des discothèques, qui resteront fermées jusqu'à début janvier, elles pourront bénéficier du dispositif " coût fixe " à hauteur de $100 \%$ pour les 4 semaines de fermeture, de la prorogation du dispositif d'activité partielle sans reste à charge et de l'exonération de charges. Par ailleurs, d'autres dispositifs continuent d'être activés : les Prêts garantis par l'État (jusqu'en juin 2022), le fonds de transition (possibilité d'apport en fonds propres) jusqu'à fin 2021, I'aide "fermeture » ouverte sous certains critères aux entreprises interdites d'accueil au public en 2021 ou dépendant à $80 \%$ d'un lieu interdit d'accueil.

\section{तépenses de santé/Hôpital/ Assurance maladie}

Un rapport de la Cour des Comptes critique la politique de prévention

Un rapport de la Cour des Comptes sur la politique de prévention en santé évalue l'efficacité de celle-ci dans 3 grandes pathologies (cancer, maladies neuro-cardio-vasculaires et diabète). Elles mobilisent plus du quart des dépenses 
totales de l'Assurance maladie (50 Md€), avec $15 \mathrm{Md} €$ de dépenses de prévention, ce qui représente un effort comparable à celui observé au Royaume-Uni et en Allemagne, mais avec des résultats décevants au regard du nombre de personnes qui en bénéficient. Selon la Cour, la responsabilité en incombe à la fois aux insuffisances du pilotage de cette politique, tant au niveau national qu'à celui des ARS, ainsi qu'à I'organisation des soins primaires en France et à leur mode de rémunération, qui souffre de la segmentation des acteurs en dépit des réformes intervenues ces dernières années (ex. communautés professionnelles de territoire de santé, contrats locaux de santé, maisons de santé professionnelles, expérimentations de rémunération au forfait). La Cour propose 4 orientations : renforcer l'efficacité de la politique de prévention et sa déclinaison opérationnelle ; lutter plus résolument contre les facteurs de risque (tabagie, alcool, obésité) ; augmenter les approches de prévention dans les pratiques professionnelles; mieux utiliser les données de santé.

\section{Une note de la Cour des Comptes sur la politique de de santé}

Dans le cadre de ses « notes sur les grands enjeux structurels pour la France ", la Cour des Comptes a publié une note sur la politique de santé et la situation de l'Assurance maladie. En 2019, les dépenses de santé (270 Md€) ont représenté 11,1\% du PIB (niveau seulement dépassé par l'Allemagne et la Suisse), dont $80 \%$ sont pris en charge par l'Assurance maladie, les complémentaires couvrant $23 \%$ des dépenses, ce qui conduit à un reste à charge pour les patients de l'ordre de $7 \%$, soit le taux le plus faible d'Europe. Deux facteurs structurels menacent la pérennité financière de l'Assurance maladie : le vieillissement de la population et l'importance croissante des pathologies chroniques, prises en charge à $100 \%$ dans le cadre de I'ALD. II s'ensuit que le régime de l'Assurance maladie, qui a pourtant bénéficié de la plus grande partie de la hausse des prélèvements obligatoires et qui avait retrouvé un quasi équilibre en 2019, va connaître durablement des déficits qui vont stagner autour de 14 Md€ à partir de 2024. Cette situation est le résultat de la prolongation de la crise sanitaire qui a occasionné des dépenses supplémentaires et des pertes de recettes considérables, d'autre part des mesures du Ségur de la Santé (+13 Md€ de dépenses par rapport à l'avant crise). La Cour propose 4 grandes orientations : 1) mettre en place une meilleure organisation du système de santé vi- sant à garantir l'accessibilité, la continuité et la qualité des soins, notamment grâce à une offre de soins de premier recours assurée par des professionnels ayant une formation de durée intermédiaire (ex. infirmiers de pratique avancée), et en rationalisant le réseau des établissements de santé ; 2 ) réformer les rémunérations des acteurs du système de santé (industries du médicament et des matériels médicaux, professionnels de santé dont les rémunérations devraient moins reposer sur le paiement à l'acte -qui favorise la hausse de la consommation- et plus sur des forfaits; 3 ) réduire les causes de dépenses qui peuvent être évitées, ce qui pose la question de l'efficacité de la politique de prévention et de la maîtrise de certaines dépenses (ex. indemnités journalières d'arrêt de travail) et du contrôle d'un certain nombre de remboursements; 4) mobiliser les technologies numériques (ex. dossier médical partagé, téléconsultation).

\section{Les propositions de la Fédération hospita- lière de France pour la politique de santé}

Dans la perspective de l'élection présidentielle, la Fédération hospitalière de France va interpeller les candidats sur la base de 30 propositions («Ambition Santé 2022 »), dont elle vient de rendre public les 4 premiers axes: 1) Un pacte pour l'accès aux soins de tous, avec en particulier un encadrement des possibilités d'installation ; 2) Une loi de programmation en santé, qui prévoirait notamment un taux plancher de l'évolution annuelle de l'ONDAM (+2,4\%) pendant les 5 prochaines années et la sécurisation des enveloppes fléchées; 3) la reconstitution des viviers de recrutement pour toutes les professions de santé, avec un effort massif pour le grand âge (100 000 recrutements) ; 4) une loi d'orientation pour le grand âge et l'autonomie, qui, outre le renforcement des recrutements, prévoit une diversification de l'offre au profit du maintien à domicile et une simplification des dispositifs de pilotage et de gouvernance des établissements.

\section{Retraites}

\section{Le Président de la République réoriente les perspectives de réforme des retraites}

Dans son entretien à TF1 le 15 décembre, Emmanuel Macron a rappelé la nécessité de reprendre le chantier de la réforme des retraites dès 2022, en insistant sur le fait qu'il faudrait travailler plus longtemps, "sans que cela signifie la même réalité pour tous ». Paraissant abandonner l'idée de "Régime universel», il a 
envisagé de remplacer les 42 régimes actuels par " trois grands régimes " pour les salariés du privé, pour le secteur public et pour les indépendants.

\section{$\lambda$ Assurance chômage}

La réforme de l'Assurance chômage définitivement validée

Dans sa décision du 15 décembre, le Conseil d'État a définitivement validé le dernier volet de la réforme de l'assurance chômage qui restait en suspens. Après avoir rejeté, en octobre dernier, le recours visant à suspendre l'entrée en vigueur de la réforme du calcul du salaire journalier de référence qui sert de base à l'indemnisation, la Haute Juridiction a validé au fond cet aspect de la réforme qui restait et qui vise à pénaliser la "permittence», laquelle consiste à alterner les contrats de courte durée et les périodes de chômage. Cette réforme, essentielle au redressement financier de I'UNEDIC, pourrait conduire à une baisse d'indemnisation sensible de plusieurs centaines de milliers de chômeurs.

\section{$\lambda$ Formation professionnelle}

Une note de la Cour des Comptes sur l'insertion des jeunes sur le marché du travail

Dans le cadre de ses notes sur «les grands enjeux structurels pour la France ", la Cour des Comptes a publié une note relative à l'insertion des jeunes sur le marché du travail. La Cour part du constat de la persistance d'un chômage élevé des jeunes de moins de 25 ans (entre 700000 et 800000 par an), plus élevé que chez nos voisins de l'Europe du Nord; elle évalue cette politique publique, caractérisée par un foisonnement de dispositifs, au regard des réformes intervenues depuis 2016 qui ont permis un recentrage vers la formation et l'accompagnement renforcé, avec la mise en place notamment du Plan d'investissement dans les compétences (PIC), et du plan « 1 jeune, 1 solution »); celui-ci articule l'intervention des 2 opérateurs principaux Pôle Emploi et les Missions locales. Selon la Cour, les résultats de dispositifs comme la "Garantie Jeunes", "l'Accompagnement intensif jeunes -AIJ » et les contrats aidés (emplois d'avenir ») seraient assez modestes en termes d'accès à l'emploi. Elle relève 4 défis pour améliorer l'efficacité de cette politique : 1) intensifier l'action en direction des publics invisibles et des décrocheurs ( NEET»); 2 )mieux cibler les 3 grands dispositifs principaux : apprentissage, Garantie jeunes, contrats aidés (cas de l'apprentissage, qui, s'il s'est développé massivement, se concentre de plus en plus sur des jeunes de niveau égal et supérieur à bac +2) ; 3) garantir la qualité du contenu des parcours les plus intensifs avec un accompagnement approprié ; 4) améliorer la coopération entre les opérateurs, ainsi qu'entre l'État et les régions, qui pourrait déboucher sur une redistribution des compétences.

\section{FISCALITÉ ET PROCÉDURES FISCALES}

\section{Procédures fiscales et Contrôle fiscal}

Cumul des sanctions fiscales et pénales en matière de fraude à la TVA devant la CJUE

La Cour de Cassation avait, dans un arrêt du 21 octobre 2020, décidé de renvoyer une question préjudicielle concernant les dispositions du droit français qui permettent le cumul de poursuites et de sanctions pénales et fiscales en matière de fraude fiscale (Cass. Crim. 21 oct. 2020, pourvoi $\left.n^{\circ} 19-81.929\right)$. Selon l'avocat général de la CJUE, en définitive, lorsque le droit interne ne permet pas d'assurer la nécessaire correspondance entre la gravité de l'infraction, d'une part, et la sévérité de toutes les sanctions cumulées, quelle que soit leur nature, d'autre part, ce qu'il appartient à la juridiction de renvoi de déterminer, la réglementation en cause sera incompatible avec le droit de I'UE du fait de la violation de l'article 50 de la Charte des droits fondamentaux de I'UE (CJUE, aff. C 570/20 en cours) qui ne s'oppose pas à une réglementation nationale qui autorise le cumul de poursuites et de sanctions, administratives et pénales, dans des cas définis à partir de critères clairs et précis, établis par la loi et détaillés de manière appropriée par la jurisprudence. A suivre...

Précisions de la CJUE dans le domaine de la coopération administrative fiscale entre États

Par un récent arrêt (CJUE, 25 nov. 2021, Luxembourg vs. L., aff. C-437/ 19), la CJUE a clarifié sa jurisprudence dans le domaine de la coopération administrative fiscale entre États. Elle précise la notion de "pertinence vraisemblable » au sens de la directive 2011/16/UE du 15 février 2011 relative à la coopération administrative dans le domaine fiscal, dans le cas d'une demande d'informations concernant un groupe de personnes identifiables, mais non désignées 
de manière nominative et individuelle. Ainsi, la notion d'identité comprend non seulement le nom et les autres données personnelles, mais aussi un ensemble de qualités ou de caractéristiques distinctives permettant d'identifier la ou les personnes faisant l'objet de ce contrôle ou de cette enquête. Selon la CJUE, il appartient à l'autorité requérante de fournir une description aussi détaillée et complète que possible du groupe de contribuables faisant l'objet d'un contrôle ou d'une enquête, tout en expliquant les obligations fiscales spécifiques pesant sur ces personnes et expose les raisons pour lesquelles lesdites personnes sont soupçonnées.

\section{$\lambda$ Impôt sur le capital}

Les propositions du Conseil d'Analyse économique (CAE) sur les droits de succession

Selon les auteurs de cette note d'analyse, le système de taxation successoral français serait fragilisé par des dispositifs d'exonération ou d'exemption qui réduisent très significativement la progressivité de l'impôt au bénéfice des plus grandes transmissions. La note formule donc quatre recommandations pour corriger le dispositif. En premier lieu, il faudrait, selon ces auteurs, refonder l'architecture de collecte et d'utilisation des données fiscales des DMTG, en harmonisant la collecte des données sur les donations et les successions et en construisant un système d'information à la DGFiP sur la base des déclarations des notaires et des assureurs. En deuxième lieu, il faudrait, selon le CAE, calculer les droits sur la base de la somme des flux successoraux perçus par les individus tout au long de la vie. En troisième lieu, il faudrait opérer une refonte de l'assiette des droits de succession, pour éliminer ou réformer les principales "niches» (assurance-vie, régime des démembrements de propriété et exemptions Dutreil à $75 \%$ ). Enfin, il faudrait, selon cette note, garantir un capital pour tous, versé à la majorité, sur la base de la citoyenneté pour limiter les inégalités les plus extrêmes d'opportunité (CAE, Repenser l'héritage, note $n^{\circ} 69$, déc. 2021). Bref, rien de bien nouveau depuis que I'OCDE a décidé de publier un rapport relatif aux impôts sur les successions et les donations dans les États de l'OCDE (Inheritance Taxation in OECD countries, 2021, v. Repères 2021). Il faut rappeler que la France occupe la $3^{\text {e }}$ place sur le podium des États prélevant le maximum de recettes fiscales à ce titre (soit 1,3\% des recettes fiscales totales, soit 12 Mds€ en 2020). Ces préconisations, qui peuvent être source de complexités, concernent surtout des
États de I'UE qui pourraient envisager d'améliorer la structure et le fonctionnement de l'impôt sur les successions (Italie, Allemagne, Espagne). La question des droits de succession semble s'imposer ces derniers jours comme l'une des pommes de discorde fiscale de la campagne présidentielle de 2022. A suivre...

\section{$\lambda$ Impôt sur les sociétés}

\section{Amendement "Charasse " et contrôle} conjoint, des précisions du Conseil d'État

L'objectif du dispositif dit de l'amendement "Charasse » est de mettre fin à des montages à buts uniquement fiscaux et consistant à endetter des sociétés, constituées à cet effet, pour le rachat d'autres sociétés détenues par le même actionnaire (art. 223 B al. 7 du CGI). Le Conseil d'État a déjà apporté des précisions sur la notion de contrôle et plus précisément sur celle $d^{\prime}$ action de concert au sens de l'amendement "Charasse » (CE, 3e et $8^{e}$ ch. r., 15 mars 2019, SAS Mi Développement 2, Req. $n^{\circ}$ 412155). Le concert était caractérisé par la conclusion d'un pacte d'actionnaires prévoyant des interdictions temporaires de cession et traduisant la volonté des parties de conduire une politique commune centrée sur la pérennité de l'action de la société cédée, les règles de majorité prévues par les statuts de la société imposant, en outre, l'accord des associés pour la prise de décision en assemblée générale. Dans la présente affaire, le Conseil d'État a précisé les contours du second élément permettant de caractériser le contrôle conjoint, relatif à la détermination des décisions prises en assemblée générale (CE, $9^{e}$ et $10^{\mathrm{e}}$ ch. r., 6 déc. 2021, SAS Financière des Éparses, Req. n439650).

\section{Publication par I'OCDE des précisions re- latives à un impôt minimum mondial}

L'OCDE a publié des éléments de détails pour aider à la mise en œuvre de la réforme du système fiscal international, destiné à garantir que les entreprises multinationales soient prochainement soumises à un taux d'imposition minimum de $15 \%$ à partir de 2023. L'impôt minimum s'appliquera aux entreprises multinationales dont les revenus sont supérieurs à $750 \mathrm{M} €$ et devrait générer environ $150 \mathrm{Mds} \$$ de recettes fiscales mondiales supplémentaires chaque année. Selon Pascal Saint-Amans, directeur du Centre de fiscalité de l'OCDE « les règles types publiées aujourd'hui sont une pierre angulaire du développement d'une solution à deux piliers, transformant les fondements d'un accord politique conclu en octobre en règles exécu- 
toires (...) Le fait que les membres soient parvenus à un consensus sur cet ensemble détaillé et complet de règles techniques démontre leur engagement en faveur d'une solution coordonnée pour relever les défis posés par une économie de plus en plus numérisée et mondialisée ».

\section{$\lambda$ Politique fiscale}

Nouveau rapport d'information à l'Assemblée nationale sur les crypto-actifs

La commission des finances de l'Assemblée nationale avait déposé, en janvier 2019, un rapport d'information concluant les travaux de la mission d'information relative aux monnaies virtuelles (E. Woerth, P. Person, A.N., doc. parl. $n^{\circ} 1624$ - v. Repères, 2019). Dernièrement, elle vient de publier un rapport d'information sur la mise en œuvre des conclusions de la mission d'information relative aux crypto-actifs (E. Woerth, A.N., doc. parl. $n^{\circ} 4753,1^{\text {er }}$ déc. 2021). Les propositions formulées ont été satisfaites dans le cadre de la loi PACTE et de la loi de finances pour 2019 ainsi que dans les mesures réglementaires corrélatives. II reste à reconnaître, dans la loi, le potentiel économique de ces progrès technologiques. Le débat a été rouvert lors de l'examen du premier projet de loi de finances rectificative pour 2021 et de la première partie du PLF pour 2022. Des amendements ont notamment proposé de mettre en place une fiscalité incitative qui encouragerait les personnes ayant réalisé des plus-values sur actifs numériques à les réinvestir dans l'économie réelle, en vain. Au total, parmi les 27 propositions formulées, 11 ont été satisfaites et 5 sont partiellement satisfaites, soit un taux d'application de $60 \%$.

\section{$\pi$ TVA}

Accord sur une proposition de directive actualisant les règles relatives aux taux de TVA

La modernisation des règles actuelles de I'UE concernant les taux de TVA était nécessaire compte tenu de l'évolution des règles générales de TVA ces dernières années. La Commission européenne a proposé en 2018 de réformer les taux de TVA. Les nouvelles règles visent à garantir plus de flexibilité aux États pour appliquer des taux de TVA réduits et nuls. Les règles élimineront également progressivement les traitements préférentiels pour les produits nocifs pour l'environnement. Le Conseil a mis à jour et modernisé la liste des biens et services pour lesquels des taux réduits de TVA sont autorisés (annexe III de la directive TVA). Le Conseil a décidé de limiter le nombre d'articles auxquels des taux réduits pouvaient être appliqués. Une fois que le Parlement aura rendu son avis sur la proposition, le Conseil adoptera formellement la directive.

La ratification législative de l'ordonnance relative à la généralisation de la facturation électronique en matière de TVA : un cavalier budgétaire?

Si le Conseil constitutionnel, saisi par plus de 60 parlementaires, juge conforme à la Constitution la majorité des dispositions de la loi de finances pour 2022 (Cons. const., décis. $n^{\circ} 2021$ 833 DC, 28 déc. 2021, LFI 2022), il vient toutefois censurer dix dispositions comme étant des "cavaliers budgétaires». A notamment été jugé contraire à la Constitution I'article 93 de la loi de finances pour 2022 qui ratifie l'ordonnance du 15 septembre 2021 relative à la généralisation de la facturation électronique dans les transactions entre assujettis à la TVA (v. Repères, sept. 2021). La disposition législative d'habilitation a été prise en loi de finances (art. 195 L. n²020-1721 du 29 déc. 2020 de finances pour 2021), conformément à la LOLF (art. 34$11-7^{\circ}$ de la LOLF) car cela concerne la collecte et le contrôle par l'administration de la TVA et donc " des dispositions relatives à l'assiette, au taux et aux modalités de recouvrement des impositions de toute nature qui n'affectent pas l'équilibre budgétaire ", sans que le Conseil constitutionnel ne fasse valoir que cet article était étranger au domaine des lois de finances (Cons. const., décis. $n^{\circ}$ 2020-813 DC, 28 déc. 2020, LFI 2021). Selon cette disposition un projet de loi de ratification devait être déposé devant le Parlement dans un délai de trois mois à compter de la publication de cette ordonnance (JORF n0216 du 16 sept. 2021), soit le 16 décembre 2021. C'est ce qu'a fait le Gouvernement, puisque cette disposition résulte de I'adoption, en première lecture par l'Assemblée nationale, d'un amendement du Gouvernement ayant reçu un avis favorable de la commission des finances de l'Assemblée nationale. Si le Conseil constitutionnel avait déjà jugé que le champ d'une loi d'habilitation ne s'impose pas au législateur lors de l'adoption de la loi de ratification (Cons. const., décis. $n^{\circ}$ 2018-761 DC, 21 mars 2018, §74), le Conseil constitutionnel est venu élargir, par cette décision, la liste des cavaliers budgétaires censurés aux dispositions qui ont pour seul objet de ratifier une ordonnance. Cette disposition aurait très certainement eu sa place, en première partie de la loi 
de finances, aux côtés d'autres dispositions relatives aux ressources de l'État qui affectent I'équilibre budgétaire, ou en loi de finances rectificative à la même place (art. 35 LOLF). En effet, il y a quelques années, au sujet de l'article 7-I de la loi n²014-1655 du 29 décembre 2014 de finances rectificative pour 2014 ratifiant l'ordonnance $n^{\circ}$ 2013-837 du 19 septembre 2013 relative à l'adaptation du code des douanes, du CGI, du LPF et d'autres dispositions législatives fiscales et douanières applicables à Mayotte, le Conseil constitutionnel avait considéré qu'il n'y avait pas lieu de soulever d'office une question de conformité à la Constitution au sujet de cette disposition de ratification (Cons. const., décis. n²014-708 DC, 29 déc. 2014, Loi de finances rectificative pour 2014, §44) car elle était complétée par d'autres dispositions législatives. Cette déclaration de non-conformité prive d'effet la loi de ratification faisant en sorte que l'ordonnance en cause demeure non ratifiée jusqu'à l'intervention d'une nouvelle loi la ratifiant, sauf si une QPC devant le Conseil constitutionnel est portée d'ici là...

\section{$\pi$ Fiscalité écologique}

La part des biocarburants retenue pour le calcul de la TGAP en conformité à la Constitution

Après une QPC renvoyée par la Cour de cassation (Cass., com., 9 sept. 2021, pourvoi $n^{\circ}$ 21-11.995), le Conseil constitutionnel a jugé conformes à la Constitution les dispositions issues de l'article 266 quindecies du Code des douanes, relatives à la part des biocarburants prise en compte pour le calcul de la TGAP (Cons. const., décis. $n^{\circ}$ 2021-946 OPC du 19 nov. 2021, Société Pétroles de la côte basque). Après avoir rappelé l'intention du législateur lors de la rédaction de ces dispositions, le Conseil en a déduit qu'il avait retenu des critères objectifs et rationnels en rapport avec l'objet de la loi. Ceux-ci n'étant pas manifestement inappropriés, il écarte le grief tiré de la méconnaissance des principes d'égalité devant la loi et juge les dispositions conformes à la Constitution, ainsi qu'aux articles 3 et 4 de la Charte de l'environnement.

\section{Actualisation des taux de la TGAP}

La DGFIP vient d'actualiser les tarifs de la taxe générale sur les activités polluantes (TGAP - art. 266 sexies à 266 duodecies du CGI) due par les entreprises dont l'activité ou les produits sont considérés comme polluants (déchets, émissions polluantes, huiles et préparations lubrifiantes, lessives, matériaux d'extraction, etc.). Son montant et le taux applicable varient selon les catégories d'activité et de produits. Le recouvrement de la TGAP, autrefois dévolu à la DGGDI, relève désormais de la DGFiP (art. 193 L. $n^{\circ}$ 2018-1317 du 28 déc. 2018 de finances pour 2019), qui a prévu un alignement des procédures déclaratives sur celles de la TVA. Sur la base du taux de croissance annuel de l'indice des prix à la consommation hors tabacs de l'année 2020, les tarifs de la TGAP (excepté ceux relatifs aux déchets non dangereux) sont actualisés pour 2022 à partir de ceux de 2021.

\section{$\pi$ Fiscalité locale}

Rapport de la Cour des comptes sur la fiscalité locale en 2021

Après la publication du premier volet, le 30 juin dernier (v. Repères, juin 2021), la Cour des comptes a publié, le 23 novembre 2021, le second fascicule (v. Repères, nov. 2021) de son rapport sur les finances locales (art. L. 132-8 du CJF). Ce dernier s'intéresse notamment à la fiscalité locale. Le rebond de l'activité économique $(6,25 \%$ en 2021 puis $4 \%$ en 2022) bénéficierait aux collectivités locales à travers un panier de recettes désormais plus sensible à la conjoncture économique. Par ailleurs, la réforme de la fiscalité locale par la suppression de la TH a sensiblement remanié le panier fiscal des collectivités territoriales. Au niveau national, l'équilibre global du dispositif est assuré par un abondement de l'État. Au niveau local, les communes perçoivent en compensation I'ancienne part de TFPB perçue par les départements. Un dispositif de compensation est mis en place. II s'agit d'un coefficient correcteur consistant à prélever les communes dont le produit de TFPB est supérieur à la perte de la $\mathrm{TH}$ perdue pour reverser à celles qui récupèrent un produit de TFPB inférieur. Les départements, les EPCl et la ville de Paris sont compensés sous la forme d'une fraction de TVA (7,9 Mds€ en 2021). Enfin, les régions qui bénéficiaient d'une partie des frais de gestion de la TH pour financer le transfert de la formation professionnelle sont compensées par une dotation de l'État reconductible d'une année sur l'autre (293 M€). Dans le cadre du plan de relance, la loi de finances pour 2021 prévoit une réduction de 10 Mds€ d'impôts de production au profit des entreprises (v. Repères, février 2021). 
Une provision DMTO pour financer l'investissement des départements en cas de difficultés

L'Assemblée des départements de France (ADF), en congrès les 2 et 3 décembre, a dressé le bilan financier 2021 des départements et dessine des pistes pour améliorer la soutenabilité des finances publiques départementales, notamment au plan fiscal. Plusieurs études et rapports, notamment de la Cour des comptes, montrent que les finances des départements ont plutôt bien résisté à la crise sanitaire, grâce au dynamisme des DMTO. L'ADF est toutefois consciente que l'évolution des DMTO a été distincte selon les territoires ces dernières années. Il semble dès lors opportun de mener à bien le chantier de la péréquation horizontale. C'est environ 1,6 Mds€ qui, au sein d'un fonds globalisé, et à l'initiative des départements, sont désormais dévolus annuellement à la solidarité interdépartementale. Dans cette perspective, I'objectif d'une répartition plus équilibrée des ressources doit être poursuivi. Le Premier ministre a annoncé une suite favorable à la proposition de l'ADF relative à la mise en place d'une provision contracyclique, soit une mise en réserve d'un surplus de DMTO, pour permettre le financement de plans d'investissement pluriannuels dans l'hypothèse de difficultés économiques. Il est désormais dans les mains du Gouvernement de confirmer cette possibilité et la date de sa mise en œuvre effective.

Avis négatif du CFL sur un projet de décret sur la nationalisation des taxes sur l'électricité

Le Comité des finances locales (CFL), composé de parlementaires et de représentants élus des régions, des départements, des communes et de leurs groupements auxquels s'ajoutent des représentants de l'État (art. L. 1211-2 du CGCT), réuni le 30 novembre dernier, et qui a pour objet principal la défense des intérêts financiers des collectivités locales en vue d'harmoniser leur position avec celle de l'État, a pris connaissance du projet de décret réformant les taxes locales sur la consommation d'électricité et instituant une part communale et une part départementale de la taxe intérieure sur la consommation finale d'électricité, et qui a reçu un avis défavorable du CFL.

\section{MANAgEMENT PUBLIC}

\section{Fonction publique}

Covid-19 et fonction publique de l'État
La direction générale de l'administration et de la fonction publique (DGAFP) vient de mettre à jour la liste des questions-réponses concernant les « mesures relatives à la prise en compte dans la fonction publique de l'État de l'évolution de l'épidémie de Covid-19».

\section{$\pi$ Ressource documentaire}

Lutter contre les violences sexuelles et sexistes dans la fonction publique

La direction générale de l'administration et de la fonction publique vient d'élaborer un projet de guide détaillant les outils statutaires et disciplinaires à la disposition des employeurs pour mieux lutter contre les violences sexuelles et sexistes dans la fonction publique.

\section{EUROPE}

\section{Euro}

$B C E$ : maintien d'une politique monétaire accommodante

Le 16 décembre 2021, le conseil des gouverneurs de la Banque centrale a estimé que "les progrès en termes de reprise économique et vers son objectif $d^{\prime}$ inflation à moyen terme autorisent une réduction graduelle du rythme de ses achats d'actifs au cours des trimestres à venir. Mais le soutien monétaire reste nécessaire en vue de la stabilisation de l'inflation au niveau de son objectif de $2 \%$ à moyen terme ". Le taux d'intérêt des opérations principales de refinancement ainsi que ceux de la facilité de prêt marginal et de la facilité de dépôt demeurent inchangés, à respectivement $0,00 \%$, $0,25 \%$ et - 0,50\%. Une politique considérée par la presse comme accommodante voire très accommodante.

\section{Les vingt ans de l'Euro}

Le 31 décembre 2021, les ministres des finances de l'Eurogroupe ont publié une tribune conjointe pour célébrer les 20 ans de la diffusion de la monnaie numéraire en euro. Dressant un bilan favorable, ils ont notamment annoncé une révision des règles budgétaires communes.

\section{Fiscalité}

Le rapport de la Commission sur les pertes de recettes de TVA

Le 2 décembre 2021, la Commission a publié un rapport sur l'écart de TVA, c'est-à-dire les pertes de recettes dues à la fraude, l'évasion et les pratiques d'optimisation fiscale dans le do- 
maine de la TVA, les faillites et les cas d'insolvabilité, ainsi que par les erreurs de calcul et les erreurs administratives. Même si l'écart continue de régresser, les pays de l'UE ont perdu 134 Mds€ de recettes de TVA en 2019.

L'accord au Conseil sur une actualisation des taux de TVA

Le 7 décembre 2021, les ministres des finances réunis en Conseil ECOFIN ont réussi à se mettre $d^{\prime}$ accord sur de nouvelles règles sur les taux de TVA, notamment sur une mise à jour de la liste des biens et services (annexe III de la directive TVA) auxquels tous les États membres peuvent appliquer des taux réduits de TVA et sur la suppression, d'ici à 2030, de la possibilité pour les États membres d'appliquer des taux réduits et des exonérations aux biens et services jugés préjudiciables à l'environnement et aux objectifs de I'UE en matière de changement climatique.

\section{INTERNATIONAL}

\section{$\pi$ Monnaies}

Le retour de l'inflation : les réactions des Banques centrales

Le mercredi 15 décembre 2021, la Réserve fédérale a décidé de réagir plus nettement face à
I'inflation. Elle a annoncé prévoir trois hausses des taux directeurs pour 2022. Ils passeront ainsi en moyenne à $0,9 \%$ fin 2022 , puis à $1,6 \%$ fin 2023 avec deux hausses prévues dans I'année, puis à 2,1\% en 2024 avec deux hausses également et enfin $2,5 \%$ à long terme.

Aurélien BAUDU (Fiscalité et procédure fiscale),

Fabrice BIN (Europe, international),

Florent GAULLIER-CAMUS (budget de l'État et des opérateurs),

Matthieu HOUSER (finances locales), Christophe SINNASSAMY (management public), Yves TERRASSE (finances sociales), Robin DEGRON (coordination).

Retrouvez sur www.revuegfp.fr :

- I'intégralité des REPÈRES d'actualité des finances et de la gestion publiques depuis 2015

- le 15 du mois, les REPÈRES d'actualité du mois précédent. 